\title{
Quantum computation with coupled quantum dots embedded in optical microcavities
}

\author{
Xin-Qi Li ${ }^{1}$ and YiJing Yan ${ }^{2}$ \\ ${ }^{1}$ National Laboratory for Superlattices and Microstructures, Institute of Semiconductors, Chinese Academy of Sciences, \\ P. O. Box 912, Beijing 100083, China \\ ${ }^{2}$ Department of Chemistry, Hong Kong University of Science and Technology, Kowloon, Hong Kong
}

(Received 4 January 2002; published 23 April 2002)

\begin{abstract}
Based on an idea that spatial separation of charge states can enhance quantum coherence, we propose a scheme for a quantum computation with the quantum bit (qubit) constructed from two coupled quantum dots. Quantum information is stored in the electron-hole pair state with the electron and hole located in different dots, which enables the qubit state to be very long-lived. Universal quantum gates involving any pair of qubits are realized by coupling the quantum dots through the cavity photon which is a hopeful candidate for the transfer of long-range information. The operation analysis is carried out by estimating the gate time versus the decoherence time.
\end{abstract}

DOI: 10.1103/PhysRevB.65.205301

PACS number(s): 73.61.- r, 03.67.Lx, 89.70.+c

To build a practical quantum computer is a challenging task, since the computational quantum objects (the qubits) must be suffficiently isolated from the dissipative environment, precisely and conditionally manipulated, efficiently read-out and initialized, and most importantly, scalable. ${ }^{1-3}$ To date, a variety of quantum computation (QC) schemes based on some unique systems have been proposed. ${ }^{4-14} \mathrm{Al}-$ though in a number of systems the proof-of-principle has been convincingly demonstrated, great challenges exist in achieving a useful quantum computer. No single system has emerged as a clear leading candidate, each having its merits and drawbacks with respect to the requirement of scalability and fault tolerance. In regard to the scalability, the solid-state implementations should represent one of the most promising directions.

A representative possibility for solid-state QC is the statein-art technology based on quantum dots (QD's). In quantum dots, the discrete electronic charge states or spin states can be exploited to encode quantum information, and the transfer of information between qubits can be mediated, for example, by electron-electron Coulomb interaction, ${ }^{15-21}$ or by optical cavity photon or vibrational phonon as a data bus. ${ }^{22-25}$ In particular, impressive experimental progress on coherently operating and entangling charge and spin states in QD's have been reported very recently. ${ }^{26-28}$

The main drawback to encode quantum information in charge states of quantum dots is the severe decoherence. To overcome it, a possible way is to apply relatively strong laser pulses to perform ultrafast operations. ${ }^{15,19,20,26-28}$ In our recent work, ${ }^{18}$ a scheme to reduce the decoherence of charge states in quantum dots was proposed to build up a single qubit from two coupled QD's. We showed that the spatial separation of the logic states can efficiently reduce the qubit decoherence. Nevertheless, several shortcomings exist there and in some of the aforementioned QC schemes based on QD's. (i) In each qubit (two coupled QD's), one and only one excess electron is required in the conduction band. This is a challenging task within current technology. (ii) The intersubband transition with $\mathrm{THz}$ lasers is currently not a mature technology. (iii) The coupling between qubits is mediated by
Coulomb interactions, which makes it very difficult to perform conditional gate operation between any pair of qubits.

In this paper, still based on the idea by constructing a single qubit from two coupled QD's to reduce decoherence, we propose an alternative scheme to remove all of these shortcomings. In this newly proposed scheme, no excess electron is required in the qubit, and quantum information is stored in electron-hole pair state. An all-optical approach is suggested for transition between valence and conduction band states that only require the well-known available laser sources. Viewing the advantages of the cavity QED effect, the cavity photon is believed to be an ideal candidate for the transfer of information, with which conditional quantum gate operation can be performed between any pair of qubits. In the proposed structure the quantum measurement of the qubit states can be achieved also optically through the quantum state holography. Specifically, the amplitude and phase information of the qubit state (i.e., the electron-hole pair state), can be extracted through mixing it with a reference state generated in the same system by an additional delayed laser pulse and detecting the total time- and frequency-integrated fluorescence as a function of the delay time. ${ }^{29,30}$

The physical system we are concerned with for quantum computation is similar as that proposed by Imamoglū et al., ${ }^{22,23}$ i.e., many QD's are located in an optical microcavity. Both the QD's and cavity are three-dimensionally (3D) confined; however, the cavity has a size and thus the fundamental wavelength much larger than the individual QD. In our structure, we suggest to use two weakly coupled QD's to construct a qubit as shown in Fig. 1. We assume a relatively large distance between neighboring qubits such that the qubits can be selectively addressed by lasers, and the Coulomb correlations between them can be neglected. As have mentioned above, in our structure no excess single electron is required in the conduction band. The quantum information is stored in electron-hole pair state: the qubit logic states $|\widetilde{0}\rangle$ and $|\widetilde{1}\rangle$ correspond to the ground state and an electron-hole pair state, respectively. (Here the tilde is used to distinguish the notation of logic states from the cavity states.) A key idea of this work is to create the electron-hole pair state with 


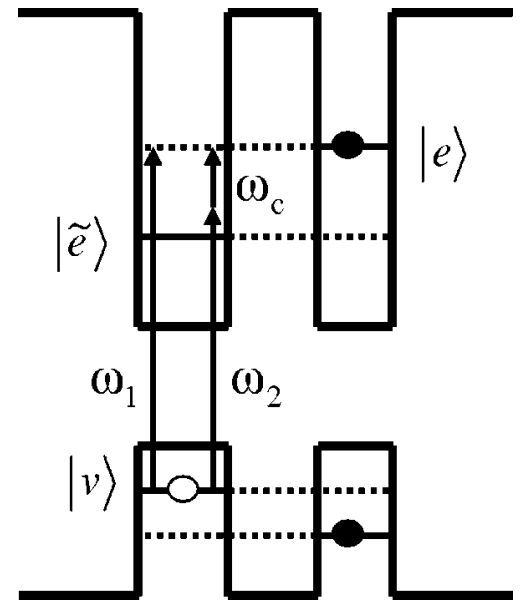

FIG. 1. Schematic diagram for a qubit constructed from two coupled quantum dots with different sizes. The plotted states are resulted from the HOMO and LUMO of the individual quantum dots. The ground state denoted by $|v\rangle$ is used for the qubit logic state $|\widetilde{0}\rangle$, and the excited state $|e\rangle$ for the logic $|\widetilde{1}\rangle$. $|\widetilde{e}\rangle$ plays a role of intermediate state with virtual occupation in the qubit operation with two photon participation. The optical coupling between states are due to the classical lasers with frequencies $\omega_{1}$ and $\omega_{2}$, and cavity photon with frequency $\omega_{c}$.

electron and hole locating largely in different dots of the qubit, which is expected to have much longer lifetime than its counterpart in a single dot. In the following, we detail two means of qubit transition as shown in Fig. 1: one involves the cavity-photon participation; another does not. As will be shown later, the cavity-photon can play a data-bus role to couple any pair of qubits.

The relevant electronic states for optical transition are shown in Fig. 1 by $|e\rangle,|\tilde{e}\rangle$, and $|v\rangle$, respectively, which are resulted by accounting for weak coupling from the HOMO and LUMO states of the two individual QD's with different sizes. Due to the confinement, we assume no intermediate levels between the two lowest conduction band states and between the highest valence band states. In general, in the absence of magnetic field, both the HOMO and LUMO levels are spin degenerate. However, in the present proposal, we exploit the charge states rather than the spin states to store quantum information, thus the superposition and even the decoherence of spin states are irrelevant to the logic states. For this reason, the spin index is omitted in the state notations. We first consider the qubit operation involving no cavity photon participation. In this case, by turning on a travelling-wave laser field with frequency $\omega_{1}$ on resonance with the energy difference between $|e\rangle$ and $|v\rangle$, the Rabi transition takes place under the interaction

$$
H_{I}^{(1)}=\Omega_{1}\left[|e\rangle\langle v| e^{i \phi}+\text { H.c. }\right] .
$$

Here $\Omega_{1}$ is the Rabi frequency, and $\phi$ is the laser phase. With the use of this interaction, arbitrary single-qubit operations can be performed.

Next, consider the cavity-photon involved transition. This is an essential ingredient to realize the two-qubit gate, in which the cavity photon plays a role of data-bus to control the two-qubit state evolution. Switching on a laser action with frequency $\omega_{2}=E_{e}-E_{v}-\omega_{c}$, a resonant transition from $|v\rangle$ to $|e\rangle$ takes place by involving two photons, namely, the $\omega_{2}$ laser photon and the $\omega_{c}$ cavity photon. A simple perturbation theory gives rise to

$$
H_{I}^{(2)}=\Omega_{\mathrm{eff}}\left[|e\rangle\langle v| a e^{i \phi}+\text { H.c. }\right],
$$

with $\Omega_{\text {eff }}=\Omega_{2} \Omega_{c} / \delta$. Here $\Omega_{2}$ is the optical coupling strength between $|\tilde{e}\rangle$ and $|v\rangle$ associating with the laser field, and $\Omega_{c}$ is the coupling strength between $|e\rangle$ and $|\tilde{e}\rangle$ due to the cavity field. $\delta$ is the detuning between the laser frequency and the transition energy from $|v\rangle$ and $|e\rangle$, i.e., $\delta=\omega_{2}$ $-\left(E_{\tilde{e}}^{-}-E_{v}\right)$. Note that this second-order process is mediated via the intermediate state $|\tilde{e}\rangle$. However, due to the offresonance of the laser frequency with the transition $|v\rangle$ $\rightarrow|\tilde{e}\rangle$, there is no real occupation on state $|\tilde{e}\rangle$, accordingly its relatively strong decoherence resulting from its radiative recombination with the intradot hole $|v\rangle$ is avoided. In the latter part of this paper, we will show that owing to the spatial separation of $|e\rangle$ from the states in the larger dot, the coherence of qubit state $|e\rangle$ can be essentially improved.

To realize the conditional two-bit gate such as the control NOT (CNOT), typical methods include the Cirac-Zoller (CZ) protocol, ${ }^{5}$ or the pulse technique developed in the spin QC model. ${ }^{9}$ In the proposals based on QD's in cavity, both of these two gating techniques have been employed. ${ }^{2,23,25}$ Very recently, an improved gating technique for the ion-trap QC was developed where only two electronic states are required, and the third auxiliary state in the $\mathrm{CZ}$ protocol is not needed. ${ }^{31}$ In the following, we employ this technique in our scheme with certain modification due to the only red-band pulse in our case. To make the description more transparent, we introduce the following notations: the states of the control qubit (the $j$ th one) and the target qubit (the $k$ th one) together with the cavity photon are denoted as $\left\{\left|\tilde{a}_{j} \tilde{b}_{k}\right\rangle|p\rangle\right.$ $\left.\equiv\left|\tilde{a}_{j}\right\rangle\left|\widetilde{b}_{k} ; p\right\rangle: a, b, p=0,1\right\}$, where 0 and 1 correspond to either the qubit state (i.e., logic $|\widetilde{0}\rangle \equiv|v\rangle$ and $|\widetilde{1}\rangle \equiv|e\rangle$ ), or the cavity field state with zero and one photon. Below we outline how to realize the CNOT gate.

(i) First, swap the control qubit state to the cavity photon state

$$
R_{j}(\pi, \phi)\left[\begin{array}{c}
\left|\widetilde{0}_{j} \widetilde{0}_{k}\right\rangle|0\rangle \\
\left|\widetilde{1}_{j} \widetilde{0}_{k}\right\rangle|0\rangle \\
\left|\widetilde{0}_{j} \widetilde{1}_{k}\right\rangle|0\rangle \\
\left|\widetilde{1}_{j} \widetilde{1}_{k}\right\rangle|0\rangle
\end{array}\right]=\left|\widetilde{0}_{j}\right\rangle \quad\left[\begin{array}{r}
\left|\widetilde{0}_{k} ; 0\right\rangle \\
i e^{-i \phi \mid}\left|\widetilde{0}_{k} ; 1\right\rangle \\
\left|\widetilde{1}_{k} ; 0\right\rangle \\
i e^{-i \phi \mid}\left|\widetilde{1}_{k} ; 1\right\rangle
\end{array}\right] .
$$

Hereafter the evolution operator $R_{j(k)}(\theta, \phi)$ is determined by the interaction Hamiltonian $H_{I}^{(2)}$ in terms of $R(\theta, \phi)$ $=\exp \left[i \theta / 2\left(|e\rangle\langle v| a e^{i \phi}+\right.\right.$ H.c. $\left.)\right]$, where $\theta=2 \Omega_{\text {eff }} T$ with $T$ the duration time of the laser pulse.

(ii) Now, the cavity photon can play a role of control qubit, which controls the evolution of the target qubit. A series of pulse operations on the $k$ th qubit by involving the participation of the cavity photon yield 


$$
G_{k}\left[\begin{array}{c}
\left|\widetilde{0}_{k} ; 0\right\rangle \\
\left|\widetilde{0}_{k} ; 1\right\rangle \\
\left|\widetilde{1}_{k} ; 0\right\rangle \\
\left|\widetilde{1}_{k} ; 1\right\rangle
\end{array}\right]=\left[\begin{array}{r}
\delta\left|\widetilde{0}_{k} ; 0\right\rangle \\
-\delta^{*}\left|\widetilde{1}_{k} ; 1\right\rangle \\
\delta\left|\widetilde{1}_{k} ; 0\right\rangle \\
-\delta^{*}\left|\widetilde{0}_{k} ; 1\right\rangle
\end{array}\right],
$$

where $\delta=e^{i \phi_{0}}$, and $\phi_{0}=\pi / 2 \sqrt{2}$. We see that the cavity photon has played a control role in the conditional evolution of the $k$ th qubit. In Eq. (4), $G_{k}$ constitutes a series of operations on the $k$ th qubit, $G_{k} \equiv H_{k}\left[P_{k} Z_{k}\left(\phi_{0}\right)\right] H_{k}$. Here $H_{k}$ and $Z_{k}\left(\phi_{0}\right)$ are the single-qubit Hadamard and phase transformations

$$
H_{k}=\frac{1}{\sqrt{2}}\left[\begin{array}{cc}
-1 & 1 \\
1 & 1
\end{array}\right], \quad Z_{k}\left(\phi_{0}\right)=\left[\begin{array}{cc}
e^{i \phi_{0}} & 0 \\
0 & e^{-i \phi_{0}}
\end{array}\right]
$$

In the subspace $\left\{\left|\widetilde{0}_{k} ; 0\right\rangle,\left|\widetilde{0}_{k} ; 1\right\rangle,\left|\widetilde{1}_{k} ; 0\right\rangle,\left|\widetilde{1}_{k} ; 1\right\rangle\right\}$, the operator $P_{k}$ has a diagonal form

$$
\begin{aligned}
P_{k} & \equiv R_{k}(-\pi / 2,0) R_{k}(\sqrt{2} \pi,-\pi / 2) R_{k}(\pi / 2,0) \\
& =\operatorname{diag}\left(1, e^{-i \pi / \sqrt{2}}, e^{i \pi / \sqrt{2}},-1\right) .
\end{aligned}
$$

(iii) Finally, the cavity photon state is swapped back to the qubit state by performing operation $R_{j}(\pi, \phi)\left|\widetilde{0}_{j} ; 1\right\rangle$ $=i e^{i \phi}\left|\widetilde{1}_{j} ; 0\right\rangle$, on the $j$ th qubit. After a phase gate $Z_{j}\left(-\phi_{0}\right)$ on the $j$ th qubit, the standard CNOT gate is realized

$$
U_{j k}\left[\begin{array}{c}
\left|\widetilde{0}_{j} \widetilde{0}_{k}\right\rangle|0\rangle \\
\left|\widetilde{1}_{j} \widetilde{0}_{k}\right\rangle|0\rangle \\
\left|\widetilde{0}_{j} \widetilde{1}_{k}\right\rangle|0\rangle \\
\left|\widetilde{1}_{j} \widetilde{1}_{k}\right\rangle|0\rangle
\end{array}\right]=\left[\begin{array}{c}
\left|\widetilde{0}_{j} \widetilde{0}_{k}\right\rangle|0\rangle \\
\left|\widetilde{1}_{j} \widetilde{1}_{k}\right\rangle|0\rangle \\
\left|\widetilde{0}_{j} \widetilde{1}_{k}\right\rangle|0\rangle \\
\left|\widetilde{1}_{j} \widetilde{0}_{k}\right\rangle|0\rangle
\end{array}\right],
$$

where $U_{j k}=Z_{j}\left(-\phi_{0}\right) R_{j}(\pi, \phi) G_{k} R_{j}(\pi, \phi)$.

In the remainder part of this paper, we present an analysis for the QC operation. In Ref. 18, based on a model GaAs system and disk geometry for the QD's, we have demonstrated by detailed numerical calculations that the ratio $\rho$ $=\tau_{d} / \tau_{G}$ can be enhanced remarkably by the spatial separation of the qubit states, where $\tau_{d}$ and $\tau_{G}$ are the qubit decoherence and gating time, respectively. In what follows we provide an alternative way to understand this issue in general, not specifying the concrete QD material and geometry.

In the approximation of two-level model, $|e\rangle$ and $|\tilde{e}\rangle$ come from the coupling of two isolated dot states $|d\rangle$ and $|\widetilde{d}\rangle$ with coupling strength $t$ and energy separation $\Delta=E_{d}$ $-E_{\tilde{d}}$. (For the highest two valence band state, similar treatment can be done). As a result, the eigenstates $|e\rangle$ and $|\widetilde{e}\rangle$ have eigenenergies $E_{ \pm}=\frac{1}{2}\left[\left(E_{d}+E_{\tilde{d}}\right) \pm \sqrt{\Delta^{2}+4 t^{2}}\right]$, and wave functions

$$
\begin{aligned}
& |e\rangle=\sqrt{1-\gamma}|d\rangle+\sqrt{\gamma}|\widetilde{d}\rangle, \\
& |\widetilde{e}\rangle=\sqrt{1-\gamma}|\widetilde{d}\rangle-\sqrt{\gamma}|d\rangle,
\end{aligned}
$$

where $\gamma=t^{2} /\left(\Delta^{2}+t^{2}\right)$. With this state nature in mind, we below estimate various decohence time and operation time in order.

The decoherence time of the qubit state is characterized by the relaxation time of $|e\rangle$. In our structure, the main intrinsic decoherence mechanisms come from the radiative relaxation and electron-phonon scattering. For both mechanisms, the relaxation rate can be expressed on the basis of Fermi golden rule as $W^{(j)}=(2 \pi / \hbar) \Sigma_{\mathbf{q}}\left|M_{f e}^{(j)}(q)\right|^{2} \delta\left(E_{e}-E_{f}\right.$ $-\hbar \omega_{q}$ ), where $\omega_{q}$ is the emitted photon (phonon) frequency, and $M_{f e}^{(j)}$ is the perturbative matrix element $M_{f e}^{(j)}(q)$ $=\left\langle f\left|H_{e p}^{(j)}(q)\right| e\right\rangle$. Here the index $j=1,2$ and 3, together with the final state $|f\rangle$, denote three relaxation channels, namely, the spontaneous radiation from $|e\rangle$ to $|v\rangle$ and $|\tilde{e}\rangle$, and the phonon-scattering induced relaxation from $|e\rangle$ to $|\tilde{e}\rangle$. Due to the spatial separate nature of the electronic states shown in Eq. (8), we roughly estimate that the relaxation rate of each channel would be reduced by a factor $\gamma$, in comparison with the relaxation rate in a single dot. As a consequence, the decoherence time can be considerably enhanced by $\tau_{d}$ $\simeq \tilde{\tau}_{d} / \gamma$, where $\tilde{\tau}_{d}$ is the intradot decoherence time.

The operation time is limited by the optical coupling between $|e\rangle$ and $|v\rangle$ via the external laser field, and between $|e\rangle$ and $|\tilde{e}\rangle$ via the cavity photon. For both cases, the coupling strengths can be expressed in terms of $\Omega_{1(c)}$ $=\left\langle e\left|H_{I}^{(1, c)}\right| v(\tilde{e})\right\rangle$. Similarly as above, due to the spatial separation of sate $|e\rangle$ from $|v\rangle$ and $|\tilde{e}\rangle$ as shown in Eq. (8), $\Omega_{1}$ and $\Omega_{c}$ will be reduced approximately by a factor $\sqrt{\gamma}$ in comparison with the corresponding intradot coupling strengths. From Eq. (1) and (2), the logic state flipping time [between $|\widetilde{0}\rangle$ ) and $|\widetilde{1}\rangle)$ ] is $\pi / \Omega_{1}$ or $\pi / \Omega_{\text {eff }}$, corresponding to the cavity photon involved or noninvolved transition. As a consequence, the gate ratio $\rho=\tau_{d} / \tau_{G}$ will be enhanced by a factor $\sim 1 / \sqrt{\gamma}$ due to the spatial separation of the qubit states. Note that $\gamma=t^{2} /\left(\Delta^{2}+t^{2}\right)$, which can be a considerably small factor by reducing $t$ and increasing the energy-level separation $\Delta$. A similar conclusion has been quantitatively demonstrated by numerical calculation in Ref. 18 .

We now parametrize the gate operation and decoherence times. Generally, consider each qubit consisting of two weekly coupled quantum dots with coupling strength between $|e\rangle$ and $|\tilde{e}\rangle$ (see Fig. 1) as, for example, $t$ $=0.01 \mathrm{meV}$, and energy difference $\Delta=E_{e}-E_{\tilde{e}}=10 \mathrm{meV}$ due to the distinct dot sizes. With these parameters, the spatial separation factor $\gamma=10^{-6}$. Concerning the optical coupling with the electronic states, for the intradot interband transition due to the laser pulse, we assume a coupling strength $\Omega_{2}=0.1 \mathrm{meV}$; and for the intradot state coupling with the cavity photon, a typical value of $\widetilde{\Omega}_{c}=300 \mathrm{MHz}$ is adopted here. ${ }^{22,23}$ To avoid a real occupation on the state $|\tilde{e}\rangle$, a detuning $\delta=1 \mathrm{meV}$ is assumed between the laser frequency and the energy difference between $|\tilde{e}\rangle$ and $|v\rangle$. As an approximate estimate, for the cavity-photon involved transition from $|v\rangle$ to $|e\rangle$, the effective Rabi frequency $\Omega_{\text {eff }}$ $=\Omega_{2} \Omega_{c} / \delta \simeq 30 \mathrm{KHz}$; and for the same transition in the absence of cavity photon, the Rabi frequency $\Omega_{1} \sim \sqrt{\gamma} \Omega_{2}$ 
$=10^{-4} \mathrm{meV}$. As a consequence, for single qubit operations, the operation time is of the order of hundreds nanosecond, whereas for two-qubit conditional operations, the characteristic time is determined by the Rabi frequency $\Omega_{\text {eff }}$ in terms of $\tau_{G}=\pi / \Omega_{\mathrm{eff}} \simeq 10^{-4} \mathrm{sec}$.

For the decoherence time, we note that with current technology the quantum dot is available with energy level spacing larger than $10 \mathrm{meV}$, thus we assume no other electronic states between $|e\rangle$ and $|\tilde{e}\rangle$ in our structure. As a result, the intrinsic decoherence channels would be the radiative relaxation and electron-phonon scattering from $|e\rangle$ to $|\tilde{e}\rangle$ and $|v\rangle$. For the spontaneous emission, if the quantum dot has certain geometric symmetry, it has been shown that the so called dark states can have radiative lifetime longer than microsecond..$^{32}$ Moreover, due to the CQED effect resulting from three dimensional cavity with high finesse, the spontaneous emission lifetime can be further suppressed. With these considerations, the radiative lifetime of the intradot conduction band state (e.g., radiative transition from $|\widetilde{e}\rangle$ and $|v\rangle$ ) can be longer than tens to hundreds of microsecond. ${ }^{32}$ For the decoherence due to interaction with phonons, the energy level spacing of $10 \mathrm{meV}$ between $|e\rangle$ and $|\widetilde{e}\rangle$ can be much different from the LO phonon energy, thus the phonon induced relaxation from $|e\rangle$ to $|\tilde{e}\rangle$ is determined by the acoustic phonon scattering. Following Bockelmann et al., ${ }^{33,34}$ the electron acoustic-phonon scattering rate will decrease rapidly with increasing the electronic level spacing. For example, in Ref. 23, acoustic phonon scattering time of $\sim 150 \mu$ s has been carried out for a similar energy level spacing (i.e., for $12.25 \mathrm{meV}$ ). Therefore, as an order of magnitude estimate, if we adopt an intradot relaxation time $\left(\tilde{\tau}_{d}\right)$ of tens of microsecond, the qubit decoherence time can be as long as tens of second (note that $\tau_{d}=\tilde{\tau}_{d} / \gamma \sim 10^{6} \times \tilde{\tau}_{d}$ ), owing to the spatial separation of the qubit states. Within this time scale, the single bit rotation can be performed as high as $10^{8}$ times, and the two-bit CNOT gate can be performed about $10^{4}$ times.

Other sources to decoherence include, e.g., the inhomogeneity of quantum dots and loss of cavity photons. Since the qubit is selectively addressed by lasers, the former might be overcome by individually tuning the laser frequency with the qubit states. The central challenge to realize the proposed QC scheme is the development of few-mode $\mathrm{THz}$ cavities with extremely low loss. An attractive candidate is the dielectric cavities, which is currently an intensive research field.

In the above discussion for decoherence, we have followed the conventional approach to neglect the effect of LO phonon scattering due to the large energy difference between the LO phonon and the electronic level spacing. This treatment is reasonable if the LO phonon has infinite long lifetime. It seems, however, questionable if one takes into account the finite lifetime of the LO phonons. Our calculations showed that the confined LO phonons in quantum dots have similar lifetime as in bulk materials, with the order of magnitude of picosecond. ${ }^{35}$ As a consequence, the LO phonon can induce electron relaxation even under the off-resonance condition. ${ }^{36}$ In the weak coupling limit as discussed here due

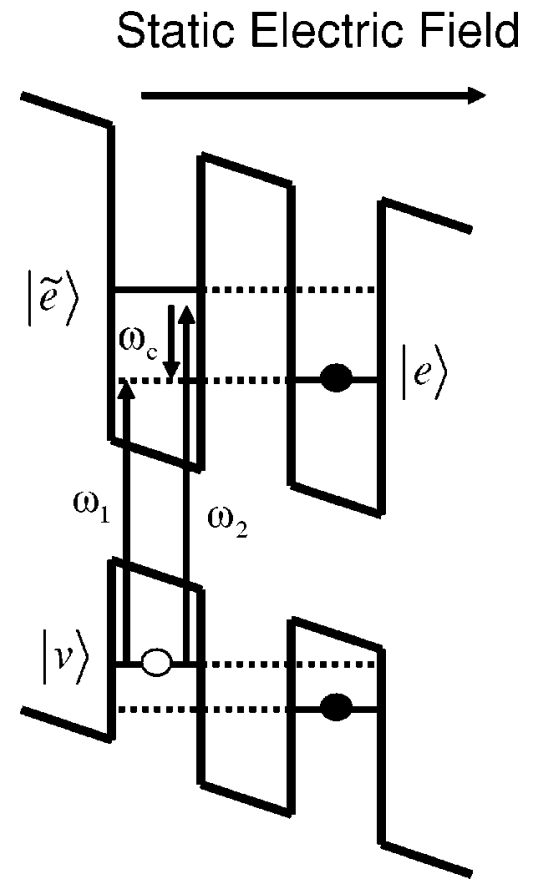

FIG. 2. An alternative configuration for qubit construction from two coupled identical QD's in the presence of external electric field. This structure is expected to be able to suppress phonon scattering from $|e\rangle$ to $|\tilde{e}\rangle$ in the low-temperature regime. The price paid here is the use of external electric field which might cause additional dephasing.

to the spatial separation of qubit states, it can be shown ${ }^{36}$ that the LO phonon induced relaxation time is proportional to $g^{-2}$, with $g$ being the coupling strength between electron and LO phonons. Since this effect is still a topic in debate in the context of phonon bottleneck in quantum dots, we are not quite sure whether it is a severe decoherence resource in the above proposed QC scheme.

To further improve the phonon scattering induced decoherence, a slightly modified qubit structure can be designed as follows. Similar as shown in Fig. 1, each qubit still constitutes two quantum dots. We suggest here to use two identical quantum dots, and to apply constantly a static electric field that results in an energy level structure as depicted in Fig. 2. In this qubit structure, the information is also stored in the states $|v\rangle$ and $|e\rangle$, and $|\tilde{e}\rangle$ plays a role of mediating transition with only virtual occupation on it. The gate operations based on this structure can be performed similarly as in the previous one, only noticing that the cavity-photon involved interaction Hamiltonian is now in the form of $H_{I}^{(2)}$ $=\Omega_{\mathrm{eff}}\left[|e\rangle\langle v| a^{\dagger} e^{i \phi}+\right.$ H.c.], instead of Eq. (2). As a result, the swap operation corresponds to generating a cavity photon via the transition from $|v\rangle$ to $|e\rangle$, and annihilating a cavity photon vice versa. The main advantage of this scheme is that the phonon scattering from $|e\rangle$ to $|\tilde{e}\rangle$ can be almost completely suppressed in the low-temperature limit. In particular, there would be no LO phonon excitations. Another merit is that the electric field can be conveniently used to tune the level spacing between $|e\rangle$ and $|\tilde{e}\rangle$ in near resonance with the cavity photon energy. The price paid here is the constant 
presence of an external electric field, whose thermal fluctuations (the Johnson noise) may cause additional dephasing. Fortunately, in our scheme the electric field is not varied to perform the logic operations. Thus the electrodes which generate the electric field can be connected to a superconducting ground, which can remove the thermal fluctuations since there is no dissipation.

In summary, we proposed a scheme based on coupled QD's embedded in optical microcavity to implement quantum computation. The proposed qubit constructed from two weakly coupled QD's is expected to have long decoherence time due to the spatial separation of the logic states. The recent progress of gating technique in ion-trap QC enables us to realize the universal quantum gate in our structure based on certain simple electronic state configuration, namely, the
LUMO and HOMO states. From the consideration on the tradeoff between phonon scattering and fluctuations of electrostatic field, we suggested two possible qubit configurations for practical choice. The most challenging aspect in the proposed QC scheme is to locate QD's in optical cavity with high finesse. Modification to the proposed gating scheme is possible by using the cavity state only as a virtual state, which can in certain sense relax the requirement to the cavity finesse.

\section{ACKNOWLEDGMENTS}

We thank the Chinese Academy of Sciences for financial support.
${ }^{1}$ D.P. DiVincenzo, Science 269, 255 (1995).

${ }^{2}$ A. Ekert and R. Josza, Rev. Mod. Phys. 68, 733 (1996).

${ }^{3}$ A.M. Steane, Rep. Prog. Phys. 61, 117 (1998).

${ }^{4}$ Q.A. Turchette, C.J. Hood, W. Lange, H. Mabuchi, and H.J. Kimble, Phys. Rev. Lett. 75, 4710 (1995).

${ }^{5}$ J.I. Cirac and P. Zoller, Phys. Rev. Lett. 74, 4091 (1995).

${ }^{6}$ D.G. Cory, A.F. Fahmy, and T.F. Havel, Proc. Natl. Acad. Sci. U.S.A. 94, 1634 (1997); N.A. Gershenfeld and I.L. Chuang, Science 275, 350 (1997);

${ }^{7}$ B.E. Kane, Nature (London) 393, 133 (1998).

${ }^{8}$ D.V. Averin, Solid State Commun. 105, 659 (1998); Y. Makhlin, G. Schön, and A. Shnirman, Nature (London) 398, 305 (1999); L.B. Ioffe, V.B. Geshkenbein, M.V. Feigelman, A.L. Fauchere, and G. Blatter, ibid. 398, 679 (1999); J.E. Mooij, T.P. Orlando, L. Levitov, L. Tian, C.H. van der Wal, and S. Lloyd, Science 285, 1036 (1999).

${ }^{9}$ D. Loss and D.P. DiVincenzo, Phys. Rev. A 57, 120 (1998).

${ }^{10}$ E. Knill, R. Laflamme, and G. Milburn, Nature (London) 409, 46 (2001)

${ }^{11}$ P.M. Platzman and M.I. Dykman, Science 284, 1967 (1999).

${ }^{12}$ A. Bertoni, P. Bordone, R. Brunetti, C. Jacoboni, and S. Reggiani, Phys. Rev. Lett. 84, 5912 (2000).

${ }^{13}$ V. Privman, I.D. Vagner, and G. Kventsel, Phys. Lett. A 239, 141 (1998).

${ }^{14}$ A.Yu. Kitaev, quant-ph/9707021 (unpublished); S. Lloyd, quant-ph/0004010 (unpublished).

${ }^{15}$ A. Barenco, D. Deustch, A. Ekert, and R. Jozsa, Phys. Rev. Lett. 74, 4083 (1995).

${ }^{16}$ G.D. Sanders, K.W. Kim, and W.C. Holton, Phys. Rev. A 60, 4146 (1999).

${ }^{17}$ T. Tanamoto, Phys. Rev. A 61, 13813 (2000).

${ }^{18}$ X.Q. Li and Y. Arakawa, Phys. Rev. A 63, 012302 (2001).

${ }^{19}$ E. Biolatti, R.C. Iotti, P. Zanardi, and F. Rossi, Phys. Rev. Lett. 85, 5647 (2000).
${ }^{20}$ L. Quiroga and N.F. Johnson, Phys. Rev. Lett. 83, 2270 (1999); J.H. Reina, L. Quiroga, and N.F. Johnson, Phys. Rev. A 62, 012305 (2000); quant-ph/0009035.

${ }^{21}$ P. Chen, C. Piermarocchi, and L.J. Sham, cond-mat/0102482 (unpublished).

${ }^{22}$ M.S. Sherwin, A. Imamoglu, and T. Montroy, Phys. Rev. A 60, 3508 (1999).

${ }^{23}$ A. Imamoglu, D.D. Awschalom, G. Burkard, D.P. DiVincenzo, D. Loss, M. Sherwin, and A. Small, Phys. Rev. Lett. 83, 4204 (1999).

${ }^{24}$ T.A. Brun and H. Wang, Phys. Rev. A 61, 032307 (2000).

${ }^{25}$ K.R. Brown, D.A. Lidar, and K.B. Whaley, Phys. Rev. A 65, 012307 (2002), quant-ph/0105102.

${ }^{26}$ G. Chen, N.H. Bonadeo, D.G. Steel, D. Gammon, D.S. Katzer, D. Park, and L.J. Sham, Science 289, 1906 (2000).

${ }^{27}$ M. Bayer, P. Hawrylak, K. Hinzer, S. Fafard, M. Korkusinski, Z.R. Wasilewski, O. Stern, and A. Forchel, Science 291, 451 (2001).

${ }^{28}$ J.A. Gupta, R. Knobel, N. Samarth, and D.D. Awschalom, Science 292, 2458 (2001).

${ }^{29}$ C. Leichtle, W.P. Schleich, I.S. Averbukh, and M. Shapiro, Phys. Rev. Lett. 80, 1418 (1995).

${ }^{30}$ T.C. Weinacht, J. Ahn, and P.H. Bucksbaum, Phys. Rev. Lett. 80, 5508 (1998); Nature (London) 397, 233 (1999).

${ }^{31}$ A.M. Childs and I.L. Chuang, Phys. Rev. A 63, 012306 (2001).

${ }^{32}$ A1.L. Efros, M. Rosen, M. Kuno, M. Nirmal, D.J. Norris, and M. Bawendi, Phys. Rev. B 54, 4843 (1996).

${ }^{33}$ U. Bockelman and G. Bastard, Phys. Rev. B 42, 8947 (1990).

${ }^{34}$ H. Benisty, C.M. Sotomayor-Torres, and C. Weisbuch, Phys. Rev. B 44, 10945 (1991).

${ }^{35}$ X.Q. Li and Y. Arakawa, Phys. Rev. B 57, 12285 (1998).

${ }^{36}$ X.Q. Li, H. Nakayama, and Y. Arakawa, Phys. Rev. B 59, 5069 (1999). 